\title{
Investigation of a multicomponent FeCoCrAITiCuMo alloy coating applied by a combined process based on atmospheric plasma metallization
}

\author{
Anvar Kadyrmetov, Dmitri Popov*, and Yevgeny Snyatkov \\ Voronezh State Forestry Engineering University named G. F. Morozov, 394087, Voronezh, Russia
}

\begin{abstract}
A brief analysis of the most common methods for producing multicomponent alloys, including high-entropy alloys (HES), is presented in terms of their use as an alternative to coatings made of traditional structural metal materials. Preliminary studies of the microstructure and phase composition of the coating obtained by plasma deposition of FeCoCrAlTiCuMo powder in the equiatomic ratio of components are presented. The results of the research showed the possibility of obtaining a multicomponent single-phase solid solution by plasma sputtering and indicated the feasibility of its further study.
\end{abstract}

\section{Introduction}

In the last decade, the properties of a new class of multicomponent high-entropy alloys (HEA) have been actively studied in metal science. Their study is associated with the need to create new, more advanced structural materials for operation in aggressive conditions caused by high temperatures and load-speed modes. The first results of HEA research were obtained by Chinese scientists, who revealed significant wear resistance and heat resistance of alloys on a nickel and iron metal matrix [1-5]. HEA contain 5 basic elements in concentrations from $5 \%$ to $35 \%$ at. and at the same time have a high entropy of mixing, which is an alloy structure stability factor from the impact of operational factors [6]. This is an advantage in comparison with traditional structural metals in terms of physical and mechanical properties, heat resistance, resistance to corrosion and wear, and other properties [7]. The properties of such alloys depend on the chemical composition and production method.

The available results of fundamental and experimental research demonstrate the unique physical and mechanical properties of HEA, which make it possible to significantly expand the scope of application of modern metallic materials [8]. The unique physical and mechanical properties of HEA led to the necessity of their use as coatings for various functional purposes.

For machine parts operating under friction and wear conditions, obtaining heat-stable wear-resistant coatings from HEA has become an urgent task. Parts operating under

\footnotetext{
*Corresponding author: qaz.7@mail.ru
} 
conditions of erosion and wear at high temperatures include parts of gas distribution mechanisms and cylinder-piston groups, internal combustion engines (valves, cylinders), brake parts, rocket engine parts (gas turbine parts).

The whole variety of possible combinations of chemical components and their concentrations in HEA has not been studied [9]; it will take more than one decade to systematize at least the main classes of multicomponent alloys. At the same time, the overwhelming number of researchers are concerned with establishing traditional links between the chemical composition, morphology of structural components and the physical and mechanical properties of the obtained materials At the same time, the development of a technology for obtaining HEA is no less valuable as a scientific and technical problem, since traditional methods do not provide a high entropy of the alloy or they are notable for a high cost. Only a few studies in the field of multicomponent alloys simultaneously affect the entire production cycle, from the choice of modifying components and determination of their concentration to the method of modification or melting. These works include, first of all, the review [10].

The overwhelming number of works is devoted to the study of all-metal samples from HEA. In relation to them, most researchers have obtained excellent results, but in practical terms, the use of HEA is difficult due to the high cost of obtaining them. At the same time, a small part of the work is aimed at researching the technology for creating multicomponent coatings with high entropy (HEA coatings). It would be advisable to use these coatings as functional coatings for the manufacture or restoration of machine parts operating under high and extreme loads. First of all, it applies to wear-resistant coatings with a thickness of $1 \ldots 5$ $\mathrm{mm}$. In this case, the parts can be made from traditional and relatively cheap alloys, on which it would be technically and economically justified to apply HEA coatings using one of the progressive methods.

Until now, such coatings were obtained mainly by expensive vacuum, ion-plasma methods, and first of all, by magnetron sputtering and vacuum-arc sputtering of chemical compounds of metals with non-metals [11], as well as by mechanical and laser alloying [12]. The use of vacuum-free technologies would reduce the cost of obtaining such coatings and increase their productivity. Such technologies include thermal gas technologies and, first of all, plasma spraying in an open atmosphere. However, such methods in relation to obtaining multicomponent coatings with high entropy have not been investigated, or insufficiently studied [13]. In this regard, it is of great scientific and practical interest to obtain HEAcoatings by combined processes based on atmospheric plasma spraying.

\section{Material and methods}

To obtain multicomponent alloys, we used a plasma technology for applying powder coatings in an open atmosphere. A mixture of metal powders FeCoCrAlTiCuMo in an equimolar ratio of components was used as materials for spraying. Coatings were applied to steel samples 45 GOST 1050-88 (rollers and prisms).

To study the chemical composition and distribution of elements over the structure, we used the functionality of a scanning electron microscope (SEM). The phase composition of the coating was determined with an ARL X'TRA X-ray diffractometer. The analysis of the microstructure of the coatings was also carried out using an optical microscope after etching the surface of thin sections with an aqueous solution of nitric acid (20\%). The mechanical properties of the phase components were assessed by the PMT-3 microhardness tester, and the integral hardness by theTK-2M (according to Rockwell).

The development of the technology for the creation of HEA coatings is possible by ensuring the uniformity of mixing and homogenization of the composition during the deposition of the coating for the implementation of a high entropy of mixing and obtaining, 
in the limit, single-phase disordered solid substitution solutions. In this case, the entropy of mixing is a necessary, but not sufficient criterion for the formation of a single-phase solid solution. For the thermodynamic stability of a solid solution, a high degree of solubility of the constituent elements in each other is required. In turn, this requires that any element of the alloy can be replaced by another element with close values of electronegativity and atomic size, while maintaining the total number of constituent elements [1]. The influence of the enthalpy of mixing and the non-configurational contributions of the entropy to the Gibbs free energy should also be taken into account. Only at a certain elemental composition a disordered solution is formed, the mixing entropy of which fully compensates the influence of both positive and negative values of enthalpy [5].

The problem of obtaining a coating with uniform mixing and homogeneity of alloy components involves the study of unknown mechanisms and patterns of coating formation, as well as the influence of its factors on the structural and phase parameters, physicomechanical and tribotechnical properties of coatings, which are the criteria for HEA coatings.

The factors of the processes of obtaining HEA are the parameters of the components composition (alloy elements) and their quantity in the alloy; external environment, means, modes and frequency of thermal, thermo-mechanical and / or mechanical impact on the formed alloy, distribution over coordinates and dynamism of this impact over time.

\section{Theory}

The prerequisites for obtaining multicomponent coatings by the technology of atmospheric plasma spraying are the possibility of homogenizing the coating composition at a high cooling rate of the alloy by means of pulse modulation of the deposition power and thermomechanical treatment. Multiple melting of the coating surface can be provided by a plasma jet in combination with thermo- and electromechanical hardening. Providing the required heating and cooling rate of coatings is possible by modulating the powers of the direct and indirect plasma torch arcs [13].

Modulation by electrical parameters of arcs affects the temperature field of the sprayed surface and has two prominent space-time aspects - local and general [14].

In this case, a minimum and uniform temperature spread over the base surface can be ensured. This is confirmed by calculations for cylindrical parts when spraying along a helical line (Fig. 1). 


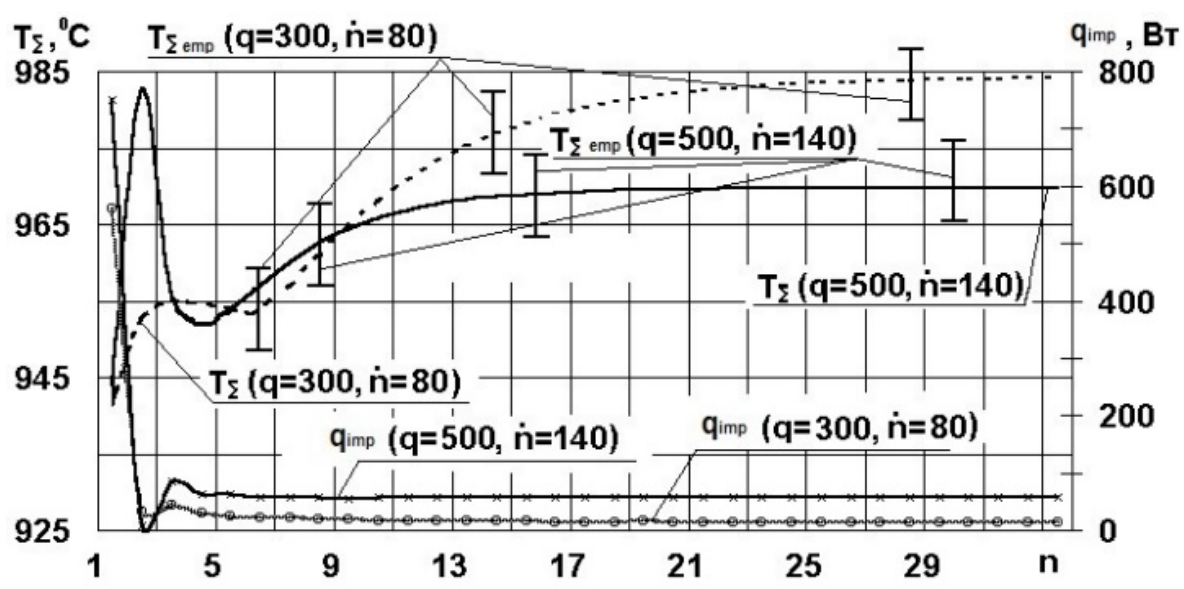

Fig. 1. Dependence of the temperature $T_{\sum}$ of the steel base on the frequency of its rotation $\dot{n}$ (rpm) and the distribution of the weighted average thermal power $q_{\text {imp }}$ when modulating the power of the external arc: $\mathrm{R}=0,04 \mathrm{~m} ; \mathrm{H}=0,002 \mathrm{~m}$; Temp - measured temperature

As can be seen from the dependence (Fig. 1) of the temperature $T_{\Sigma}$ on the values of the weighted average amplitude of the power $q_{i m p}$ and the revolutions $\dot{n}$ : the distribution of temperatures when changing the pulse power is $2 \ldots 7 \%$ of the average temperature value (except for the first loop).

The temperature spread, starting from the second turn, is noticeably lower when compared with the traditional method of plasma preheating with the exclusion of longitudinal movement of the plasmatron along the part. The spread is $10 \ldots 20 \%$ of the average value, that is, $80 \ldots 180^{\circ} \mathrm{C}$ less than when using the conventional approach. Reducing the spread of the surface temperature will reduce the levels of thermal deformations on it in certain areas of the surface, this will create conditions for more uniform chemical and phase composition and physical and mechanical properties of the coating and base.

The use of modulation of the power parameters of the remote arc when coating the profiled surfaces is even more effective. This applies to wedge-shaped parts, such as excavator bucket teeth or cultivator shares. On the surface of such parts, the uneven heating can be $600{ }^{\circ} \mathrm{C}$. The use of modulation of the parameters of the remote arc can reduce this value to $40 \ldots 60^{\circ} \mathrm{C}$.

Empirical studies made it possible to conclude that due to parameters modulation of the remote arc with direct polarity, the following increases: adhesion of the coating to the base by $1,15 \ldots 1,25$ times, microhardness - by $1,1 \ldots 1,2$ times, fatigue strength - up to 1.2 times, wear resistance of the coating $-1,25 \ldots 1,35$ times.

\section{Results}

Metallographic studies of thin sections of a coating obtained by atmospheric plasma spraying before and after chemical etching showed that its structure had a uniform fine-grained appearance, pores and cracks were practically absent (Fig. 2.) 


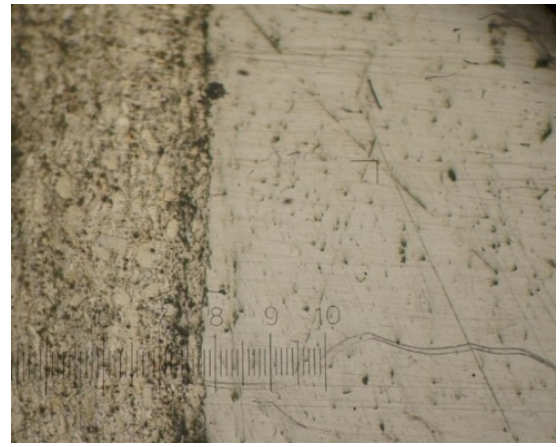

a

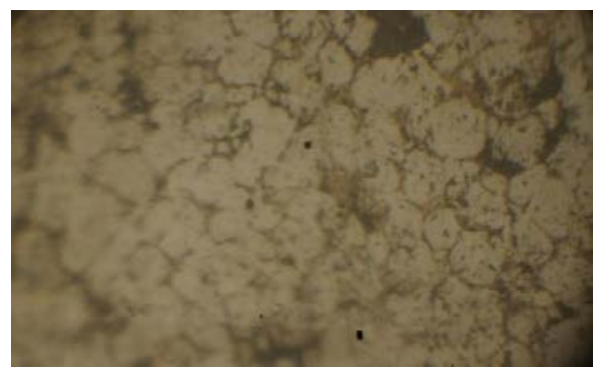

$\mathrm{c}$

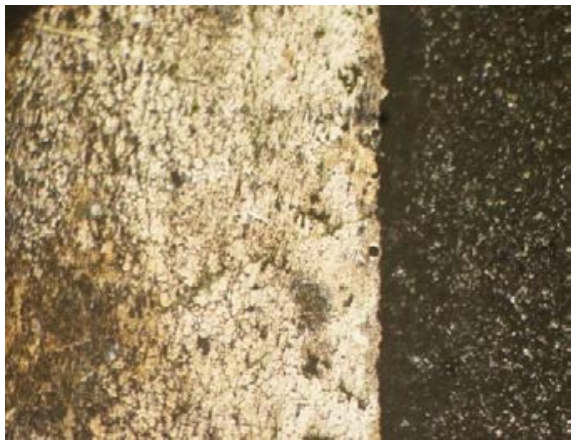

b

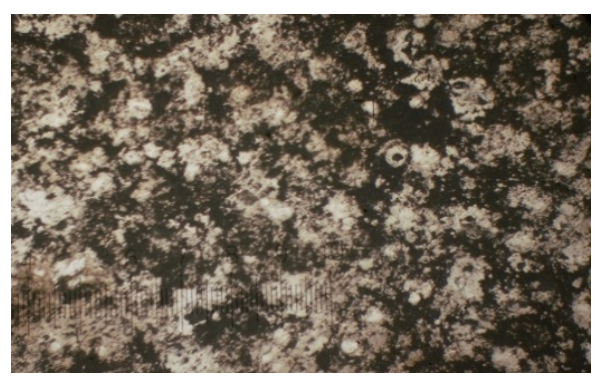

d

Fig. 2. Photo of thin sections: a - thin section not etched $\times 50$; $b$ - etched thin section $(20 \% \mathrm{H} 2 \mathrm{NO} 3) \times$ 50 ; - thin section not etched $\times 100 ; d$ - etched thin section $(20 \% \mathrm{H} 2 \mathrm{NO} 3) \times 100$;

The coating had high chemical resistance to oxidizing agent (H2NO3), this can be seen in the photo (b), where the interface between the base (on the right - steel 45) and the coating (on the left) is clearly marked, the steel was deeply etched and became almost black, while only the grain boundaries were marked on the coating. At a higher resolution, the grain boundaries were observed in Fig. 2 (photo. c, d). As a result of metallographic observations, it was noted that the structure of the coating had mainly two metallic phases, one of which was located along the boundaries of the other (the main one in terms of quantitative ratio). The hardness of the coating was 47 HRC.

As a result of observations of a characteristic area of the coating on a scanning electron microscope (SEM), a layered structure was revealed in Fig. 3 (a) and a map of the distribution of atoms of the coating components in Fig. 3 (b, c, d). From the observations of the images, it can be seen that individual elements, such as $\mathrm{Mo}, \mathrm{Cu}, \mathrm{Ni}$, were distributed relatively uniformly over the coating structure Fig. 3 (b), while $\mathrm{Ti}, \mathrm{Cr}, \mathrm{Fe}, \mathrm{Co}, \mathrm{Al}$ were slightly dissolved in the structure Fig. 3 (c, d), forming expressed conglomerates. The weak solubility of these elements is associated, in our opinion, with a relatively higher melting point, low diffusion of atoms of the elements. The results of the observations (SEM) indicate a low ability of individual metals in a mixture of powders to form a solid single-phase solution under these thermodynamic conditions. 


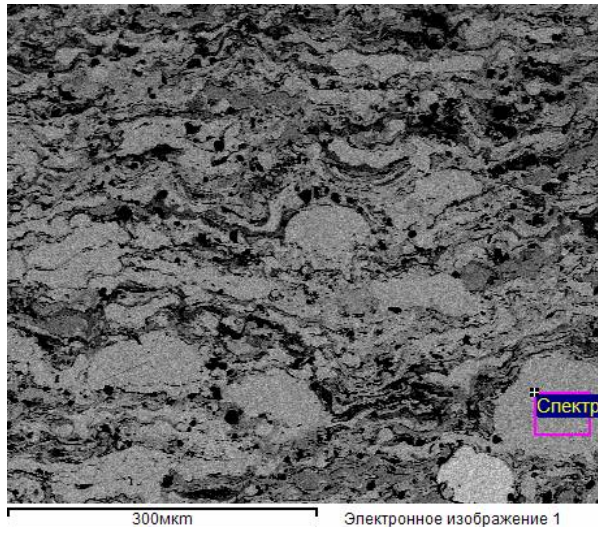

a

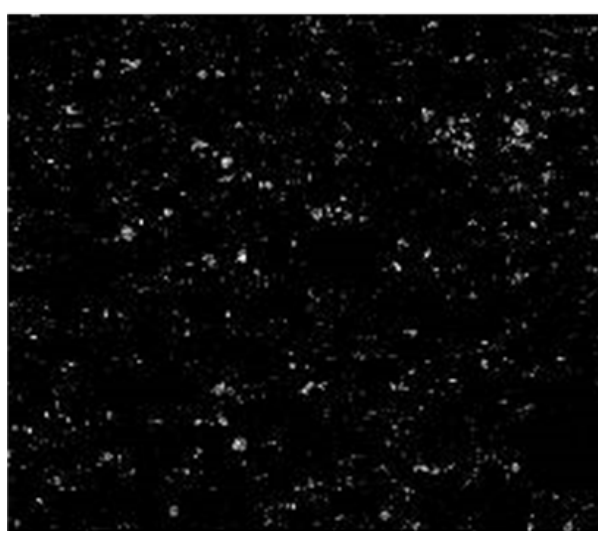

$\mathrm{c}-\mathrm{Ti}$

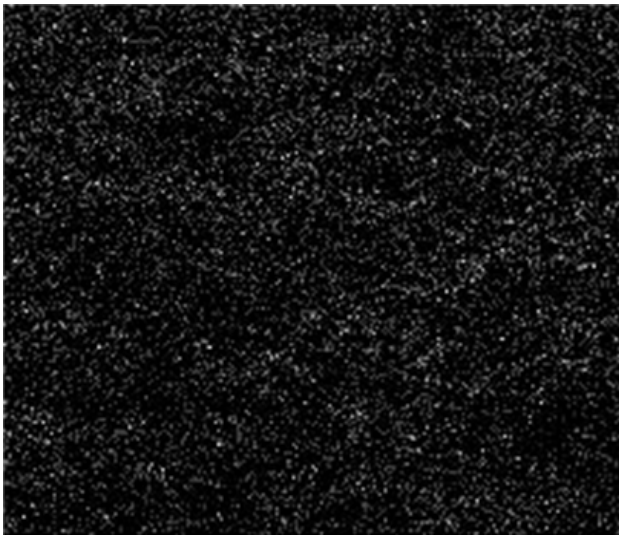

$\mathrm{b}-\mathrm{Mo}$

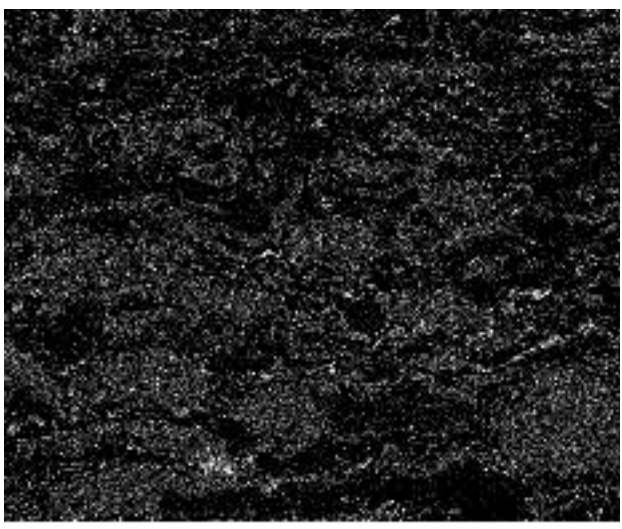

$\mathrm{d}-\mathrm{Cu}$

Fig. 3. Images (SEM): a - coating structure; b, c, d - map of distribution of elements $\mathrm{Mo}, \mathrm{Ti}, \mathrm{Cu}$, respectively, over the surface area (a)

To study the phase composition of the coating, an X-ray analysis was carried out, the results of which are shown in Fig. 4. Decoding of the X-ray diffraction pattern showed that the main phase of the coating is a solid solution of $\mathrm{Ni}-\mathrm{Cu}$ with a 50/50 atomic ratio of atoms with an face-centered crystal lattice (lattice parameter 3,563677216064889). Based on the data obtained, we can conclude that the coating is a $\mathrm{Ni}-\mathrm{Cu}$ alloy in which the atoms that make up the alloy have formed an interstitial solid solution, and can also be located along the grain boundaries, i.e. to be there in nanoscale clusters and not be detected by X-ray diffractometry.

There was no nickel in the composition of the powder mixture, its appearance in the alloy is explained by the fact that, before applying the basic composition, a substrate was created from the self-fluxing chromium-nickel alloy (designation in Russian «ПР-HX17CP4») powder, the basis of which is Ni (73.8\% of the mass). During the deposition of the basic composition of the powder, the substrate melted and a generalized $\mathrm{Ni}-\mathrm{Cu}$ melt was formed. 


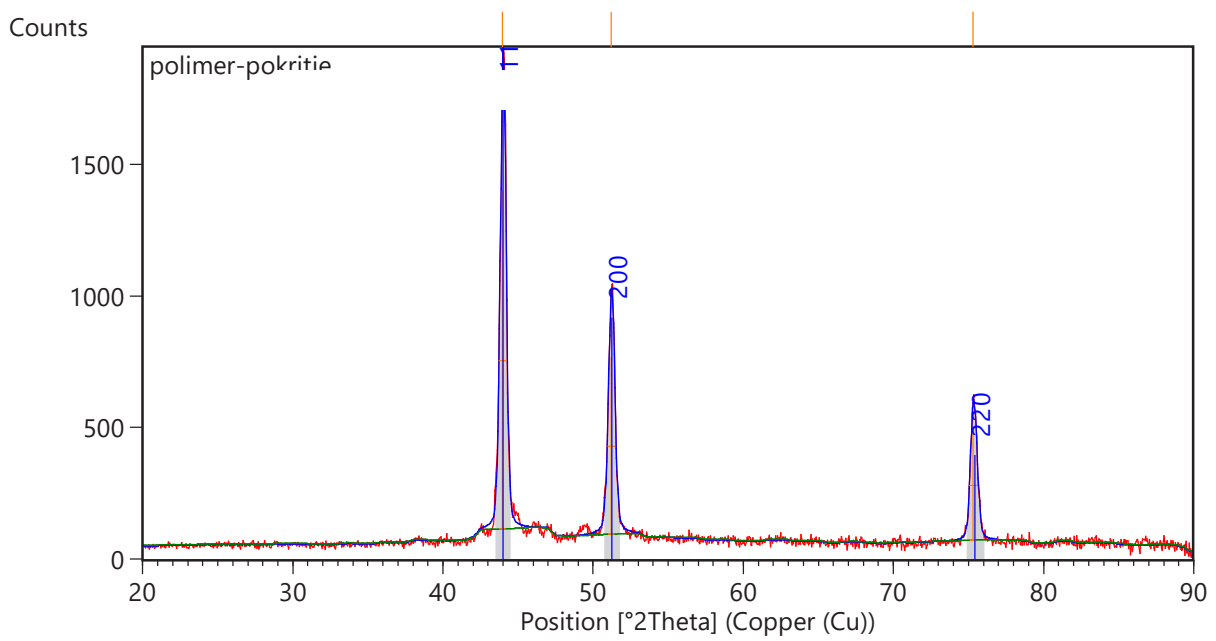

Fig. 4 Diffraction pattern obtained from a sample of FeCoCrAlTiCuMo coating in an equimolar ratio of components

\section{Discussion and conclusions}

The results of the studies carried out on the FeCoCrAlTiCuMo multicomponent alloy in the equiatomic ratio of the components obtained by atmospheric plasma spraying showed the possibility of obtaining a single-phase solid solution using the plasma spraying technology. The disadvantages of the alloy caused by its nonuniformity in structure can be minimized by multiple melting of the coating surface, as well as by using a special design of the plasmatron, which provides a high concentration of thermal energy at the spray spot. This will significantly increase the rate of melting or melting of the sprayed coating, increase diffusion and uniform distribution of elements in the crystal structure of the coating. Thus, the obtained results confirm the assumption of the possibility of obtaining single-phase multicomponent alloys by plasma spraying and indicate the expediency of further research with the possibility of introducing this technology in the manufacture and repair of transport vehicles. The priority areas for further research on plasma spraying technology in the development of multicomponent alloys are:

- the use of multiple reflow in an inert environment;

- selection of chemical elements compiling the alloy, which have high solubility in each other (with a close serial number);

- to ensure the formation of a predominantly body-centered phase, which gives the alloys higher mechanical properties;

- provision of high heating power and coating cooling rate up to $106-107 \mathrm{~K} \cdot \mathrm{s}-1$, followed by quenching to create a fine-grained structure;

- investigation of the effect of mechanical strengthening of the coating on its wear resistance and prevention of fatigue cracks on the working surface of parts. It is advisable to consider electromechanical action as one of the methods of such processing. 


\section{References}

1. B. Cantor, I. T. H. Chang, P. Knight, A. J. B. Vincent, Microstructural development in equiatomic multicomponent alloys, Materials Science and Engineering: A : Elsevier, 375, pp. $213-218$ (2004)

2. F. Otto, The influences of temperature and microstructure on the tensile properties of a CoCrFeMnNi high-entropy alloy / F. Otto, A. Dlouhy, H. Bei, Acta Material, Vol. 61, pp. 2628-2638 (2013)

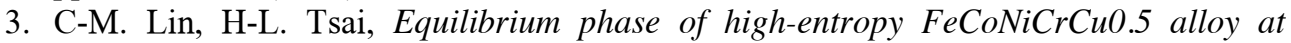
elevated temperature, J Alloys Compd., Vol. 489, pp. 619-622 (2010)

4. M-R. Chen, S-J. Lin, J-W. Yeh, M-H. Chuang, S-K. Chen, Y-S. Huang, Effect of vanadium addition on the microstructure, hardness, and wear resistance of AlO.5CoCrCuFeNi high-entropy alloy, Metall Mater Trans, Vol. A 37(5), pp. 1363-1369 (2006)

5. A. V. Kuznetsov, D. G. Shaysultanov, N. D. Stepanov, G. A. Salishchev, O. N. Senkov, Tensile properties of an AlCrCuNiFeCo high-entropy alloy in as-cast and wrought conditions, Materials Science and Engineering, Vol. 533, pp. 107-118 (2012)

6. J. W. Yeh, Recent progress in high-entropy alloys, Annales de Chimie Science des Material, Vol. 31, pp. 633-648 (2006)

7. M. V. Karpets, O. M. Maliuchenko, Properties of a multicomponent high-entropy alloy AlCrFeCoNi doped with copper, Problems of friction and wear, Vol. 2., pp. 103-111 (2004)

8. Y. Zhang, T.T. Zuo, Z. Tang, M.C. Gao, K. A. Dahmen, P.K. Liaw, Z-P. Lu, Microstructures and properties of high-entropy alloys, Progress in Materials Science, Vol. 61, pp. 1-93 (2014)

9. Z. P. Lu, H. Wang, M. W. Chen, I. Baker, J. W. Yeh, An assessment on the future development of high-entropy alloys: Summary from a recent workshop, Intermetallic, Vol. 66, pp. 67-76 (2015)

10. A. D. Pogrebnjak, A. A. Bagdasaryan, I. V. Yakushchenko, V. M. Beresnev, The structure and properties of high-entropy alloys and nitride coatings based on them, Russian Chemical Reviews, Rev. 83 (11), pp. 1027-1061 (2014)

11. S. A. Firstov, Superhard coatings from high-entropy alloys, Science and innovation, Vol. 9, pp. 32-39 (2013)

12. B. He, The Phase Evolution and Property of FeCoCrNiAlTix High-Entropy Alloying Coatings on Q253 via Laser Cladding, Coatings, Vol.7, pp. 157 (2017)

13. A. M. Kadyrmetov, D. A. Popov, A. V. Vikulin, V. I. Voronetsky, R. V. Stegantsev Prospects of obtaining multicomponent coatings by atmospheric plasma spraying, Voronezh scientific and technical Bulletin, Vol. 4 (26), pp. 46-54 (2018)

14. A. M. Kadyrmetov, S. N. Sharifullin, A. S. Pustovalov, Mathematical modeling of plasma deposition and hardening of coatings-switched electrical parameters, Journal of Physics: Conference Series 669 012052: VII Conference on Low Temperature Plasma in the Processes of Functional Coating Preparation IOP Publishing, pp. 1-5 (2016) 